\title{
Erratum to: Impact of damaging and recovery on the temperature dependence of the work function of oxide electrodes in fluorescent lamps ${ }^{\star}$
}

Eur. Phys. J. Appl. Phys. 96, 11301 (2021). https://doi.org/10.1051/epjap/2021210149

Reinhard Langer ${ }^{1,2, *, * *}$, Irina Paul ${ }^{1,2, *, * * *}$, and Reinhard Tidecks ${ }^{1}$

${ }^{1}$ Institut für Physik, Lehrstuhl für Experimentalphysik II, Universität Augsburg, Universitätsstraße 1, 86159 Augsburg, Germany

${ }^{2}$ LEDVANCE GmbH (former part of OSRAM AG), Berliner Allee 65, 86136 Augsburg, Germany

Received: 9 December 2021

- In equation (11) a factor "T" has to be added at the end of the equation.

- In the title of Section 3.7.3 "fowler" should be replaced by "Fowler".

- In the first line of Section 3.7.3 "stae" should be replaced by "state".

- In equation (27) the exponent of the term in brackets has to be corrected to be " $+3 / 2$ ".

- On the right side of equation (49) in the first term a factor $\Phi_{0}$ has to be added.

The authors thank Claus Müller to draw the attention on their printing errors. We apologize for the errors made.

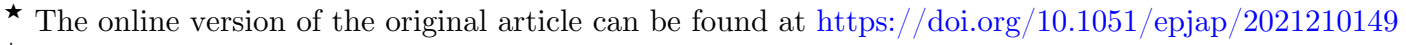

* e-mail: reinhard.langer-physik@t-online.de

** Present address: Deutsches Patent- und Markenamt, Zweibrückenstraße 12, D-80331 München, Germany. This article represents the author's personal opinion and not that of the German Patent and Trademark Office.

*** Present address: Instrument Systems Optische Messtechnik GmbH, Kastenbauerstraße 2, 81677 Munich, Germany.
} 